\title{
Targeted delivery of a novel anticancer compound anisomelic acid using chitosan- coated porous silica nanorods for enhancing the apoptotic effect
}

\author{
Rajendran Senthilkumar, Didem Sen Karaman, Preethy Paul, Emma Johansson, Magnus \\ Odén, John E. Eriksson and Jessica M. Rosenholm
}

\section{Linköping University Post Print}

\section{Tweet}

N.B.: When citing this work, cite the original article.

Original Publication:

Rajendran Senthilkumar, Didem Sen Karaman, Preethy Paul, Emma Johansson, Magnus Odén, John E. Eriksson and Jessica M. Rosenholm, Targeted delivery of a novel anticancer compound anisomelic acid using chitosan-coated porous silica nanorods for enhancing the apoptotic effect, 2015, BIOMATERIALS SCIENCE, (3), 1, 103-111.

http://dx.doi.org/10.1039/c4bm00278d

Copyright: Royal Society of Chemistry

http://www.rsc.org/

Postprint available at: Linköping University Electronic Press http://urn.kb.se/resolve?urn=urn:nbn:se:liu:diva-113162 


\section{Biomaterials Science}

\section{ARTICLE}

Cite this: DOI: 10.1039/x0xx00000x

Received 00th January 2014, Accepted 00th January 2014

DOI: $10.1039 / \times 0 \times x 00000 x$

www.rsc.org/

\section{Targeted delivery of a new anticancer compound Anisomelic acid using chitosan-coated porous silica nanorods for an enhanced apoptotic effect in vitro}

\author{
Rajendran Senthilkumar ${ }^{a}$, Didem Şen Karaman ${ }^{b}$, Preethy Paul ${ }^{a}$, Emma M. Björk $^{c}$, \\ Magnus Odén ${ }^{c}$, John E. Eriksson ${ }^{*, a}$ and Jessica M. Rosenholm ${ }^{*}, b$
}

Targeted cancer therapies are currently a strong focus in biomedical research. The most common approach is to use nanocarrier-based targeting to specifically deliver conventional anticancer drugs to enhance their therapeutic efficacy, increase bioavailability, and decrease the side-effects on normal cells. A step further towards higher specificity and efficacy would be to employ specific novel drugs along with specific nanocarrier-based targeting. Our recent studies have demonstrated that a plant-derived diterpenoid compound, anisomelic acid (AA), induces apoptosis in cervical cancer cells. In this work, we describe the development of a folic acid (FA)-targeted AA delivery system using chitosan-coated rod-shaped mesoporous silica particles (Chitosan-NR-MSP). The cellular internalization and and uptake enhancement of the FA-Chitosan-NR-MSP towards cancerous folate receptor (FR) -positive (SiHa and HeLa) and/or normal FR-negative (HEK 293) cells were assessed, which indicated that the intracellular uptake of FA-conjugated Chitosan-NR-MSP was more target-specific. Furthermore, the induction of apoptosis by AA-loaded chitosan-coated rod-shaped particles on SiHa cells was studied. By employing caspase activation and PARP cleavage as measure of apoptosis, the FA-particle mediated AA treatment was clearly more effective, significantly enhancing apoptosis in comparison to non-targeted Chitosan-NR-MSP or free AA in SiHa cells, suggesting that the FA-Chitosan-NR-MSPs can be potentially utilized as a drug delivery system for cervical cancer treatment.

\section{Introduction}

Cancer is a generic term for a broad group of diseases, involving uncontrolled growth and spread of abnormal cells. In the continuing search for agents that may treat or ameliorate the affiliation of cancer, natural products have provided an endless supply of active compounds that are increasingly being exploited. In many cases, this quest has already resulted in leads and scaffolds for drugs ${ }^{1-3}$ and many reports highlight the use of plant-derived compounds as anticancer drugs ${ }^{4,5}$ that contribute to new leads in clinical trials. ${ }^{6}$

Anisomelic acid (AA) (Fig. S1 $\dagger$ ) is a diterpenoid compound isolated from the medicinal plant Anisomeles malabarica ${ }^{7}$, which we have previously shown to be cytotoxic to oral and cervical cancer cells. ${ }^{8}$ Recently, we have shown that AA induces apoptosis and cause cell cycle arrest in SiHa cervical cancer cells by specifically down-regulating the viral oncoproteins, E6 and E6, ${ }^{9,10}$ which are the main reasons behind cervical carcinogenesis. ${ }^{10}$

Targeted delivery of anticancer drugs using nanocarriers is a promising approach to alleviate the drawbacks of conventional cancer therapy, offering the advantages of reduced dosage of drug, and delivering it at the tumor site to avoid sideeffects. ${ }^{11,12}$ In the past decade, mesoporous silica particles (MSPs) have attracted considerable attention in related biomedical applications. ${ }^{13,14}$ Meanwhile, rod-shaped nanoparticles have also gained less but significant attention in the last few years. ${ }^{15,16}$ In line with previous studies regarding rod-shaped particles, our recent investigation on the morphology effect of spherical and rod-shaped MSPs on cellular internalization showed that the rod-shaped MSPs displayed increased cellular uptake as compared to the spherical particles. ${ }^{17}$ Thus, such studies could have important implications for the further development of MSP-based drug delivery systems.

While inorganic carrier platforms such as MSPs are newer to the drug delivery scene, their organic counterparts such as polymer-based nanomaterials have been already quite established as drug delivery systems due to their favourable characteristics, such as dispersion stability, low toxicity, ease of surface modification, sustained drug release and target-specific localization. ${ }^{18}$ To add to the flexibility of both material classes, constructs from both material classes can be combined to create synergistic qualities for the carrier system design. One such easily obtainable structure is coating of inorganic nanomaterials with organic polymers. Polymers used for coating aiming at drug delivery applications must be biocompatible and biodegradable. Among natural polymers, polysaccharides tend to be internalized and degraded rapidly, thus enabling a 
moderate intracellular release of the drug. ${ }^{19}$ Chitosan is a natural cationic polysaccharide, obtained by alkaline deacetylation of chitin, which is derived from shrimp and other crustacean shells. In the literature, from a drug delivery point of view, chitosan is one of the few natural cationic biopolymers available and has been increasingly employed as drug carriers in the form of chitosan nanoparticles generated by employing different preparation techniques. ${ }^{20}$ In addition to this, chitosan has been implied to provide slow/controlled drug release with the advantage of $\mathrm{pH}$-responsiveness. ${ }^{21,22}$ This inherent property of chitosan can lead to prolonged retention time of drugs and continuous drug release in vivo as well as improving the drug bioavailability. Chitosan has also been utilized in improving the solubility, stability and efficacy of drugs. ${ }^{23}$ These accumulated literature data consequently endow chitosan with advantages that could be beneficial for different biomedical and drug delivery applications. ${ }^{24}$

In the present study, we aimed to design and evaluate the applicability of a drug carrier, which possesses the combined advantages of both rod-shaped mesoporous silica nanoparticles (NR-MSPs) and chitosan in order to deliver the potential anticancer compound AA with increased efficacy over free AA to cervical cancer cells. In light of the considerations above, we envisioned that elongated nanoparticles would be more favourable for therapeutic applications based on targetingspecificity, biodistribution, as well as cellular internalization profiles. ${ }^{17,25,26}$ In addition to the morphological advantages of NR-MSP, the prepared particle design also comprises the advantages of chitosan, which was electrostatically adsorbed on the NR-MSPs after drug loading. This course-of-action resulted in that chitosan also provided better dispersability for the drugloaded NR-MSPs and could further function as a capping agent for the pores of NR-MSPs by achieving coating on the particle surfaces $^{27}$ in addition to providing the system with the known pH-responsive property of chitosan. Furthermore, we also aimed at uptake enhancement by means of active cellular targeting for the delivery of AA to folate receptor (FR) enriched cancer cell surfaces by conjugating folic acid (FA) to chitosan, ${ }^{28}$ with subsequent coating of the NR-MSP surfaces with the folic acid-chitosan conjugate. Additionally, we investigated the in vitro cytocompatibility and cellular uptake of these Chitosan-NR-MSPs in FR-positive cervical cancer cell lines (HeLa and SiHa). We report that the apoptotic effects of anisomelic acid-loaded Chitosan-NR-MSPs in SiHa cancer cells clearly demonstrate that particle-mediated delivery of AA is significantly enhanced over free AA, and that FA-targeted Chitosan-NR-MSPs showed further increased efficacy in the targeted cell lines as compared to non-targeted Chitosan-NRMSPs.

\section{Experimental}

\section{Materials}

Tetraethyl orthosilicate (TEOS) (reagent grade, 98\%), triblock copolymer $\mathrm{EO}_{20} \mathrm{PO}_{70} \mathrm{EO}_{20} \quad(\mathrm{P} 123)$ and heptane $(99 \%$, ReagentPlus), Chitosan (low molecular weight), 1-ethyl-3-(3dimethylaminopropyl) carbodiimide) (EDC), N-hydroxy succinimide (NHS) and Cyclohexane (anhydrous, 99,5\%) were purchased from Sigma-Aldrich. Hydrochloric acid (purity $>37 \%$, puriss.), Ammonium fluoride (purity > 98.0\% puriss) and Folic Acid (> 97\% HPLC) were obtained from Fluka, Germany. Cell proliferation reagent WST-1 was purchased from Roche Diagnostics GmbH, Germany. Anisomelic acid
(AA) was isolated from Anisomeles malabarica. ${ }^{10}$ Milli-Q water $(18.2 \Omega)$ was used throughout the study.

\section{Preparation of fluorescently labeled rod-shaped mesoporous silica particles}

Rod-shaped mesoporous silica particles (NR-MSP) were synthesized according to the literature procedure. ${ }^{29}$ Briefly, TEOS was used as silica source and block-co-polymer P123 was the employed pore structure directing agent in this synthesis. The synthesis was performed under acidic conditions ( $\mathrm{HCl})$ with the presence of $\mathrm{NH}_{4} \mathrm{~F}$ and heptane in the reaction solution. The synthesis solution consisted of the molar ratio $1 \mathrm{P} 123 / 1.8 \mathrm{NH}_{4} \mathrm{~F} / 280 \mathrm{Heptane} / 60 \mathrm{TEOS} / 356 \mathrm{HCl} / 10335 \mathrm{H}_{2} \mathrm{O}$. After calcination, the produced NR-MSPs were subsequently labeled by post-synthesis grafting with fluorescein isothiocyanate (FITC) - modified aminopropyltriethoxysilane silane (APTES) in toluene. The modification of APTES was carried out by pre-reacting FITC with APTES in $2 \mathrm{~mL}$ acetone with a molar ratio of $1: 3$ and stirring for $2 \mathrm{~h}$ under inert atmosphere.

\section{Anisomelic acid loading, surface coating and characterization of rod-shaped mesoporous silica particles}

Anisomelic acid loading to the rod-shaped mesoporous silica particles (NR-MSP) was carried out by soaking $20 \mathrm{mg}$ of NRMSPs into different concentrations of $10 \mathrm{~mL} \mathrm{AA-cyclohexane}$ solution and keeping the suspension stirring overnight at room temperature. After adsorption of AA, the AA loaded NR-MSP samples (NR-MSP/AA) were centrifuged and the obtained NRMSP/AA precipitates were vacuum dried. Afterwards, AA elution study was carried out in order to plot the AA adsorption isotherm as a function of equilibrium concentration and to investigate the highest Anisomelic acid (AA) loading amount. The details of AA elution from NR-MSP is described in the Supporting Information part and the adsorption isotherm is presented in Fig. $\mathrm{S} 2 \uparrow$.

The surface coating of vacuum dried NR-MSP/AA was carried out by electrostatic adsorption of chitosan in 2-(Nmorpholino) ethanesulfonic acid (MES) buffer solution (10mM, $\mathrm{pH}$ 5.5) after determination of the highest AA loading amount for NR-MSP/AA. Chitosan adsorption was preferred for modification of NR-MSP and NR-MSP/AA samples in order to enhance the dispersability and stability of particles in suspension while capping the pores of NR-MSPs after drug loading.

Folic acid conjugated chitosan-coated AA loaded rodshaped mesoporous silica particles (FA-Chitosan-NRMSP/AA) were prepared for potential targeted delivery of AA. The conjugation of folic acid with chitosan was proceeded as follows: ${ }^{28}$ Chitosan was dissolved in $1 \mathrm{v} / \mathrm{v} \%$ acetic acid aqueous solution at concentration of $5 \mathrm{mg} / \mathrm{mL}$. After complete dissolution of chitosan, the solution was filtered and the $\mathrm{pH}$ was adjusted to 5.5 with $0.1 \mathrm{M} \mathrm{NaOH}$, and then diluted with MES buffer solution to a $1 \mathrm{mg} \mathrm{mL}^{-1}$ concentration. As a first step of folic acid conjugation with chitosan, 1-ethyl-3-(3dimethylaminopropyl) carbodiimide) (EDC) cross-linker was reacted with the carboxylic acid groups of folic acid in ethanol (at a molar ratio of 1:1) at room temperature and stirred continuously until a well-dissolved solution was obtained, containing the amine-reactive intermediate $\mathrm{O}$-acylisourea. In order to increase the yield of EDC-mediated amine coupling, N-hydroxy succinimide (NHS) was used as stabilizer for the amine-reactive intermediate in MES buffer. ${ }^{30,31}$ The stabilization of amine-reactive intermediate was provided with 
the addition of N-hydroxy succinimide (NHS) at a molar ratio 1:1 in MES buffer and kept stirring for 20 minutes. The stabilized amine-reactive folic acid solution was then mixed with the chitosan solution at a molar ratio of 1:0.16 between chitosan and folic acid. This mixture was stirred at room temperature for $4 \mathrm{~h}$. After that, the $\mathrm{pH}$ of the reaction solution was increased from 5.5 to 9 with $1.0 \mathrm{M} \mathrm{NaOH}$, and the solution was centrifuged. The purification of the folic acid-chitosan conjugate was carried out by dialysis of the precipitate obtained from the last centrifuge step. After a 3 day dialysis step, the sample was freeze-dried and coated onto NR-MSP and NRMSP/AA samples.

The success of folate conjugation on the chitosan polymer was confirmed with a NanoDrop 2000c UV-Vis Spectrophotometer by dissolving folic acid-conjugated chitosan in MES buffer and reading the absorption maximum intensity of folate at $285 \mathrm{~nm}$. With the help of obtained results from this part of the study, the mass of folate per mg of FA-ChitosanNR-MSP was estimated by combining the result obtained for the FA-Chitosan amount on NR-MSP surfaces, which was determined with a TGA-Netzsch STA 449F1 Jupiter instrument. The TG resolution of this instrument is $0.025 \mu \mathrm{g}$ and the sample amounts were about $10 \mathrm{mg}$. TGA was carried out under air atmosphere and in aluminum crucibles, at the

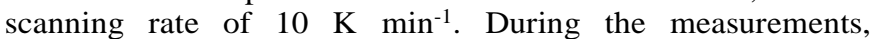
thermograms were recorded within the range of $30-550{ }^{\circ} \mathrm{C}$ and the results were analyzed with the help of software Proteus 5.

In addition to all detailed characterization in our previous study, ${ }^{17}$ the mesoscopic ordering and morphology of NR-MSP was investigated with a Scanning Electron Microscope (SEM) as well as a Transmission Electron Microscope (TEM), the latter being carried out with a FEI Tecnai G2 TF 20 UT microscope operated at $200 \mathrm{kV}$. Redispersibility and zeta potential of the particles were investigated in MES buffer at $\mathrm{pH}$ 5.5 with a Malvern ZetaSizer NanoZS (please see the Supporting Information part for the nomenclature of all the prepared samples).

\section{In vitro release kinetic studies of Anisomelic acid (AA)}

Drug release profiles and the $\mathrm{pH}$ responsiveness of the drug delivery system were investigated for the AA loaded NR-MSP (NR-MSP/AA) and Chitosan coated AA loaded NR-MSP (Chitosan-NR-MSP/AA) samples. The release of AA from Chitosan- NR-MSP and NR-MSP was followed at two different $\mathrm{pHs}$, of which one was mimicking endosomal $(\mathrm{pH} 5.5)$ and the other normal extracellular $\mathrm{pH}(\mathrm{pH} 7.2)$ at $36^{\circ} \mathrm{C}$ for 30 hours. $^{21}$ Accurately weighed NR-MSP/AA and Chitosan-NR-MSP/AA samples were dispersed in $\mathrm{pH} 7.2$ (HEPES) buffer and $\mathrm{pH} 5.5$ buffer (MES) solutions with a concentration of $200 \mu \mathrm{g} \mathrm{mL}^{-1}$. For this part of the study, Spectra/Por ${ }^{\circledR}$ Float-A-Lyzer ${ }^{\circledR}$ G2, Ready-to-Use laboratory dialysis devices were used, and at different time points the whole sample volumes of $6 \mathrm{ml}$ were removed, and replaced with a new $6 \mathrm{~mL}$ of fresh medium. As a final step of the release study, the aliquots were analyzed for AA concentrations by using UV spectroscopy reading at a wavelength of $230 \mathrm{~nm}$ after preparing AA standard curves. Samples of each time points were measured 3 times and the average value of the measurements were used to plot the release curves. In the analysis step, the NR-MSP and Chitosan-NRMSP samples were employed as blanks for each time points after treating them the same as actual samples.

\section{Cell culture}

The human cervical cancer cell lines, SiHa, HeLa and HeK 293 cells were obtained from the American Type Culture Collection (ATCC) and cells were cultured in Dulbecco's modified Eagle's medium (Sigma) supplemented with $10 \%$ fetal calf serum (BioClear), 2mM L-glutamin, 100 units $\mathrm{mL}^{-1}$ penicillin, $100 \mu \mathrm{g} \mathrm{mL}^{-1}$ streptomycin. Cell growth was carried out in a humidified atmosphere at $37^{\circ} \mathrm{C}$ in $5 \% \mathrm{CO}_{2}$.

\section{WST-1 Cell viability assay}

The WST-1 cell proliferation assay is based on the cleavage of the tetrazolium salt WST-1 to formazan by mitochondrial dehydrogenases of viable cells. Cells $(\mathrm{SiHa} / \mathrm{HeLa})$ were seeded in a 96-well culture plates at about $10^{3}$ cells well ${ }^{-1}$ and cultured until $70 \%$ confluence. The cells on the 96-well plates were then incubated with various concentrations of NR-MSP (in

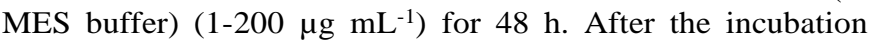
period, $10 \mu \mathrm{L}$ of the WST-1 reagent was added to each well, and the plate was incubated again for $1-2 \mathrm{~h}$ at $37^{\circ} \mathrm{C}$ in $5 \% \mathrm{CO}_{2}$. After 1-2 hr. incubation with WST-1 reagent, the plate was stirred thoroughly for $30 \mathrm{sec}$. on a shaker and the absorbance of the colored formazan at $440 \mathrm{~nm}$ was measured using a Varioskan Microplate Reader. The WST-1 assay was repeated in triplicate.

\section{Intracellular uptake studies by flow cytometry}

Cells were seeded into 12-well plate and incubated for $24 \mathrm{~h}$ at $37{ }^{\circ} \mathrm{C}$. The $65-70 \%$ confluent cells were then incubated with FITC-labeled NR-MSPs at different concentrations (0.3-5 $\mu \mathrm{g} / \mathrm{mL}$ ) for 4 and $24 \mathrm{~h}$. After incubation with NR-MSPs, the cells were trypsinized and the extracellular fluorescence was quenched by resuspension in trypan blue $\left(200 \mathrm{mg} \mathrm{mL}^{-1}\right)$ for 5 $10 \mathrm{~min}$ at room temperature. Thereafter, the cells were washed and resuspended in $0.5 \mathrm{~mL}$ phosphate-buffered saline (PBS), and analyzed using a FACS Calibur flow cytometer. All measurements were carried out in triplicate and each experiment, 10,000 events per samples were recorded.

\section{Uptake studies by confocal scanning microscopy}

SiHa, HeLa and Hek 293 cells were seeded in 12-well plates containing cover slips, one day before treatment, and the cells were treated with a fixed concentration of $1 \mu \mathrm{M}$ of ChitosanNR-MSP and FA-Chitosan-NR-MSP in growth medium for 4 and/or $24 \mathrm{~h}$. After the treatment, the cells cultured on cover slips were washed with PBS and fixed with $3 \%$ paraformaldehyde for $5 \mathrm{~min}$ at room temperature, and mounted with DAPI (Vectashield; Vector Laboratories Inc, Burlingame, CA). The intracellular localization of NR-MSPs were viewed and imaged under a confocal laser scanning microscope (LSM 510 META, Carl Zeiss Inc., Thornwood, NY) equipped with an argon laser using FITC filter.

\section{Caspase activity assay}

Apoptotic cells were determined by caspase-3 PE staining. The PE active caspase- 3 apoptosis assay kit (BD Pharmingen, San Diego, CA) was used according to the manufacture's protocol. In brief, after reaching 80-90\% confluency, $\mathrm{SiHa}$ cells were treated with $20 \mu \mathrm{M}$ of AA or chitosan coated AA loaded NRMSP and FA-Chitosan coated AA loaded NR-MSP incubated for $24 \mathrm{~h}$. After the incubation time, both floating and adherent cells were collected, washed with cold PBS, and fixed with 
Fix/Perm buffer for 20 minutes on ice. The cells were subsequently washed twice with Perm/Wash buffer and resuspended to the same buffer containing $10 \mu \mathrm{L}$ antibody. After 30 minutes incubation at room temperature, the cells were washed and resuspended to Perm/Wash buffer. The samples were analyzed by FACS Calibur flow cytometry (FL-2, FSC channel) using Cell Quest software. All experiments were performed in triplicate.
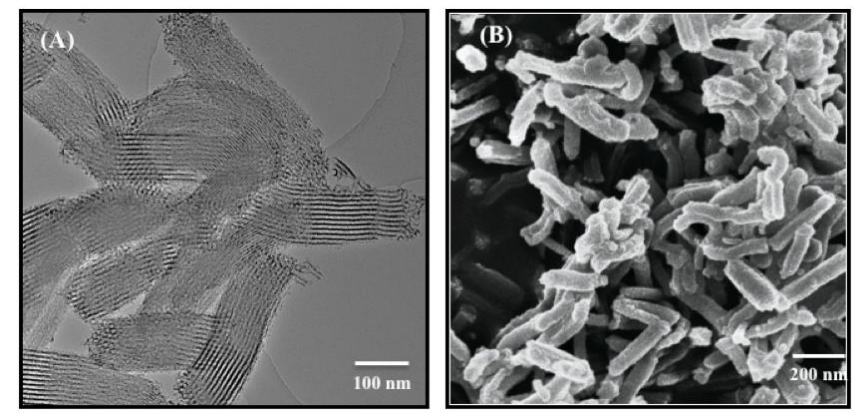

Fig. 1 Transmission electron microscopy (TEM) (A) and scanning electron microscopy (SEM) (B) images of the produced rod-shaped mesoporous silica particles (NR-MSP).

Table 1. Polydispersity index (PDI) and net surface charge $(\zeta$ potential) of NR-MSPs in MES buffer (10 mM, pH 5.5).

\begin{tabular}{lcc}
\hline Samples & PDI & Zeta Potential [mV] \\
\hline NR-MSP & 0.1 & -16 \\
Chitosan-NR-MSP & 0.2 & 32 \\
FA-Chitosan-NR-MSP & 0.1 & 29 \\
NR-MSP/AA & 0.1 & -43 \\
Chitosan-NR-MSP/AA & 0.2 & 33 \\
FA-Chitosan-NR-MSP/AA & 0.1 & 25 \\
\hline
\end{tabular}

\section{Western blot analysis}

SiHa cells were incubated with Chitosan-NR-MSP/AA and FAChitosan-NR-MSP/AA samples corresponding to $20 \mu \mathrm{M}$ AA for $24 \mathrm{~h}$. After $24 \mathrm{~h}$ incubation, cells were lysed with Laemmli sample buffer 32 and the cell lysates were heated at $95{ }^{\circ} \mathrm{C}$ for 10 min after which proteins were separated by 12.5 or $15 \%$ SDSPAGE and transferred to polyvinylidene difluoride membranes (Millipore Corporation, USA). The membranes were blocked with $5 \%$ non-fat milk, then incubated with primary antibodies such as as poly-(ADP-ribose) polymerase (PARP) (clone C-210; Sigma-Aldrich), caspase 3 (Cell Signaling Technology, Davers, MA, USA), anti-FLOR1 (FR $\alpha)$ antibody (clone EPR4708(2); Lifespan BioSciences) and $\beta$-actin (clone AC-40; Sigma-Aldrich) overnight on shaker at $4{ }^{\circ}$ C.Proteins were detected using the ECL method (Amersham Biosciences) on Xray film after incubation with the appropriate horseradish peroxidase-conjugated secondary antibodies for $1 \mathrm{~h}$ at room temperature. Each Western blot result is representative of at least three independent experiments.

\section{Results and discussion}

\section{Characterization of rod-shaped mesoporous silica particles (NR-MSP)}

Detailed characterization of the pristine NR-MSPs has already been carried out in our previous study. ${ }^{17}$ With regard of the pore structural characterization from this study, the pristine NR-MSPs possess a pore size of $11.3 \mathrm{~nm}$ (by DFT) with a unit cell parameter of $14.8 \mathrm{~nm}$. In the presented electron microscopy images (Fig. 1), the mesoscopic ordering (TEM) and morphology (SEM) of NR-MSPs is further confirmed, revealing rod-shaped porous particles with an aspect ratio (AR) of about 4 (width 500 $\mathrm{nm}$ height $125 \mathrm{~nm}$ ) and pores aligned along the major axis of the rod-shaped particle body.

Examination of the hydrodynamic size and zeta potential values of uncoated (pristine) and coated NR-MSPs (ChitosanNR-MSP and FA-Chitosan-NR-MSP particles, before and after drug loading) showed that both the chitosan-coated and uncoated particles are fully redispersible, thus resulting in low polydispersity index (PDI) values. The main difference in the surface characteristics of these particles were observed in the zeta potential values (Table 1), which is an indication of the success of the electrostatic adsorption of chitosan onto NRMSPs.

In addition to the change in zeta potential after chitosan and FA-Chitosan coating on NR-MSP, further confirmation was carried out by the determination of the adsorbed FA-Chitosan amount (in weight percent with respect to the whole particle system). For this analysis, thermogravimetry was used and the adsorbed FA-Chitosan was calculated as about $10 \mathrm{wt} \%$ (as illustrated in Fig. S3†). In order to determine the potential of further use of chitosan as a pore-capping agent, the surface excess value of FA-Chitosan was calculated after coating the polymer on the particles. In order to extract this value, the external surface area of NR-MSP was deduced from the $t$-plot analysis from the nitrogen sorption measurement, Fig. S4†. The surface excess value of FA-Chitosan for NR-MSP was estimated by combining the values form TGA and $t$-plot analysis. According to these calculations, a surface excess value of approximately $25 \mathrm{nmol} \mathrm{m}^{-2}\left(3 \mathrm{mg} \mathrm{m}^{-2}\right)$ was obtained. From the existing studies in the literature, the chitosan conformation was thus considered as a mingled conformation of trains, loops, tails or coils after adsorption to the particle surface, which can provide very sufficient capping of the loaded AA molecules in the pores of the particles. ${ }^{27}$

By combining the UV-Vis spectrophotometric result of folate amount per mass of FA-Chitosan with the thermogravimetric result of FA-Chitosan amount in weight percent with respect to the total particle mass, the folate amount was thus determined as approximately $0.25 \mu \mathrm{g}$ per $1 \mathrm{mg}$ of FAChitosan-NR-MSP.

\section{Drug Release from NR-MSP and Chitosan-NR-MSP}

In this novel particle design, which has been schematically depicted in Fig. S5†, AA was loaded into the pores of rodshaped mesoporous silica particles (NR-MSP) instead of conventional spherical-shaped particles. The drug loading amount after surface coating was calculated as $130 \mu \mathrm{g} \mathrm{mg}^{-1}$ (13 wt $\%$ ) by elution of AA from Chitosan-NR-MSP/AA particles into ethanol, and this value was considered for all apoptosis studies. The kinetic drug release profiles determined under sink conditions (Fig. 2) indicated that the chitosan coating induced a sustained release to the carrier system, which was 
dependent on the surrounding $\mathrm{pH}$. Much higher degree of burst release was observed for the NR-MSP/AA as compared to Chitosan-NR-MSP/AA at both acidic and neutral $\mathrm{pH}$. Also for the non-chitosan-coated carrier system, AA release reached a plateau for the NR-MSP/AA in the first 2 hours at $\mathrm{pH} 5.5$ whereas this duration was 6 hours at $\mathrm{pH} 7.2$. However, this may be a result of varying AA solubility at different $\mathrm{pHs}$, which might also affect the determination of drug concentration by spectroscopic techniques. Nevertheless, a clear difference in burst-effect was observed for Chitosan-NR-MSP/AA, which thus seems to provide sufficient capping of the drug and consequently significantly suppress the degree of burst release. Also, the drug retention provided by the chitosan coating was more efficient at neutral $\mathrm{pH}$ than acidic, well matching the criteria of drug being retained in extracellular conditions to avoid premature release.

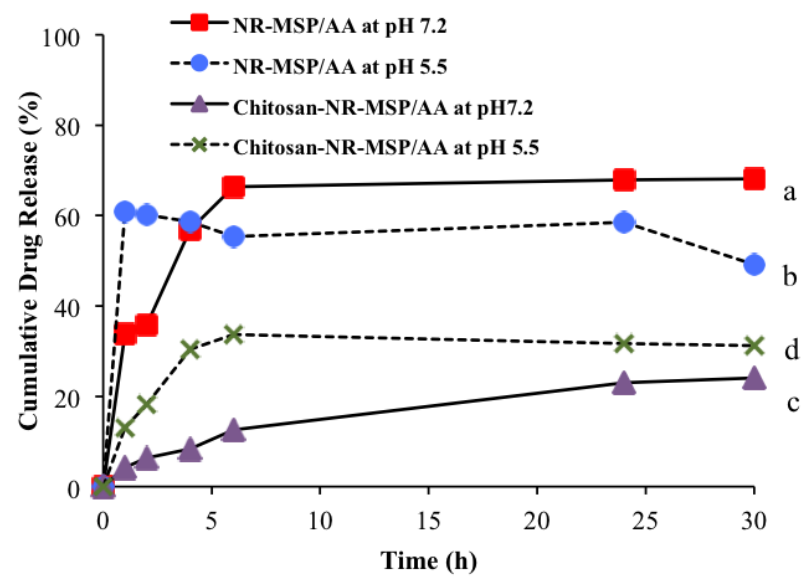

Fig. 2 AA release from NR-MSP a) at $\mathrm{pH}: 7.2$, b) at $\mathrm{pH}: 5.5$, Chitosan- coated NR-MSP c) at $\mathrm{pH}: 7.2$ and d) at $\mathrm{pH}: 5.5$.

The pH-responsiveness of the chitosan coating for the developed drug carrier was not as drastic as that observed for similar systems in the literature, in which the release of ibuprofen from MSPs was modified by covalent bonding of chitosan to the particle surface, whereby the ibuprofen release was approximately $90 \%$ at $\mathrm{pH} 5$ and $20 \%$ at $\mathrm{pH} 7.4 .^{21}$

In our study, the reason not to observe such high release efficacy at $\mathrm{pH} 5.5$ might be connected to the difference in the thickness of the chitosan coating on the particle surface as compared to the abovementioned study, as well as the higher solubility of ibuprofen in aqueous solvent as compared to AA. The chitosan coating may require being degraded in order to exhibit a higher (more complete) drug release under sink conditions. On this note, it has been suggested that the in vitro release of the entrapped drug catechin from (pure) chitosan nanoparticles was increased from $32 \%$ to $60 \%$ upon enzymatic degradation of the chitosan. ${ }^{33}$ Before in vivo studies are carried out for this drug carrier design, enzymatic degradation of chitosan from the particles should be investigated, which can also be explanatory for the observed apoptosis enhancement by this design as shown in the results related with the apoptosis induction (below). Another reason not to observe complete drug release might be low water solubility of AA, which can thus also be a drawback for the determination of drug release under sink conditions.

\section{In vitro evaluations of NR-MSPs}

\section{Cell viability}

The in vitro cytocompatibility of chitosan-coated NR-MSP (Chitosan-NR-MSP) and folate-conjugated chitosan-coated NR-MSP (FA-Chitosan-NR-MSP) was examined on cultured $\mathrm{SiHa}$ and HeLa cancer cells adopting the WST-1 assay. The cells were exposed for $48 \mathrm{~h}$ to the medium containing the chitosan-coated NR-MSP in the concentration range of 0-200 $\mu \mathrm{g} \mathrm{mL}^{-1}$. MES buffer was used as the solvent control. The cell viability by Chitosan-NR-MSP and FA-Chitosan-NR-MSP was investigated in a dose-dependent manner on both $\mathrm{SiHa}$ and HeLa cells (Fig. 3). WST-1 assay results revealed that the coated NR-MSPs were not cytotoxic towards neither SiHa nor HeLa cells, but actually a slight increase in cell proliferation was observed as compared to the untreated cells. These results are in agreement with previous studies, indicating that chitosan improves the cell proliferation and induces no cytotoxic effects on the cells. ${ }^{34,35}$ These results are promising for the determination of the biocompatibility of Chitosan and FAchitosan-coated NR-MSPs, crucial for their use in vivo, even though evaluation of the complete in vivo biocompatibility picture obviously is much more complex than what can be determined using cell viability assays. ${ }^{36}$
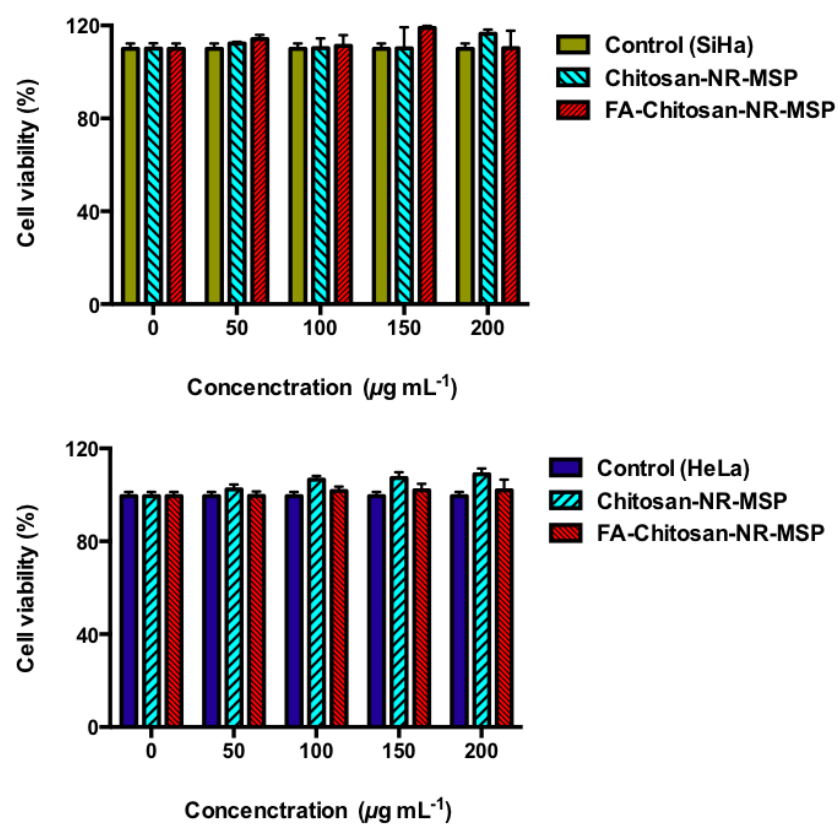

Fig. 3 In vitro dose dependent cytocompatibility of ChitosanNR-MSPs and FA-Chitosan-NR-MSP after $48 \mathrm{~h}$ incubation time on $\mathrm{SiHa}$ (A) and HeLa (B) cell lines. Cell viability was measured by the WST-1 assay.

\section{Cellular Uptake}

The cellular internalization of FITC-labeled Chitosan-NR-MSP and FA-Chitosan-NR-MSPs by human cervical cancer cells (SiHa and HeLa) and noncancerous embryonic kidney epithelial cells (Hek 293) was evaluated using flow cytometry 
(FACS) and confocal microscopy techniques. The cells were treated with various concentrations of surface coated NR-MSPs for two different time periods, $4 \mathrm{~h}$ and $24 \mathrm{~h}$, and it was observed that the uptake of NR-MSPs was increased in a concentration- and time-dependent manner (Fig. 4A and Fig. 4B). Furthermore, the FA-Chitosan-NR-MSPs showed higher uptake than the Chitosan-NR-MSPs at a lower concentration, which were then decreasing at higher concentration, suggesting that the cells were reaching their saturation capacity. ${ }^{37}$ The comparison of NR-MSPs uptake in both cervical cancer cells and epithelial cell lines at a specific concentration, i.e. $1 \mu \mathrm{g} \mathrm{mL}^{-1}$ demonstrated that, while HeLa cells express a much higher level of folate receptors ${ }^{38}$ (Fig. S6 $\dagger$ ), they internalized much more FA-Chitosan-NR-MSP $(\sim 90 \%)$, as compared to SiHa cells (Fig. 4C). In contrast, the embryonic kidney epithelial HeK 293 cell line, which lacks abundant folate receptors, showed lower FA-Chitosan-NR-MSP uptake compared to the cervical cancer cell lines, which can be even more clearly seen when the mean fluorescence intensity (MFI) values were measured for the all three cell types (Fig. 4D). The total cellular uptake of the FA-targeted particles thus followed the same order as the FR expression (Fig. S6 $\dagger$ ), i.e. HeLa > $\mathrm{SiHa}>\mathrm{HeK}$ 293. Consequently, the uptake of the presented carrier system can be enhanced by using FA as targeting ligand.
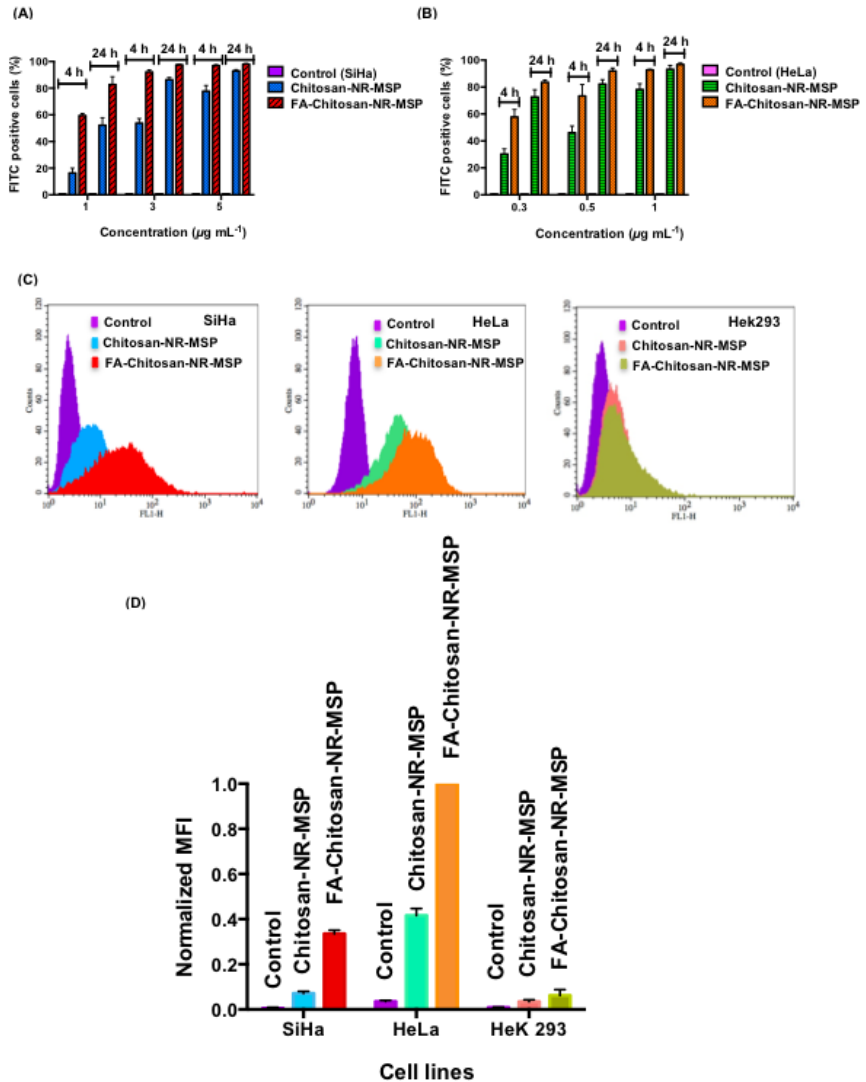

Fig. 4 Cellular uptake of Chitosan-NR-MSP and FA-ChitosanNR-MSP in (A) SiHa and (B) HeLa cell lines measured by Flow cytometry. (C) Histogram profiles of cervical ( $\mathrm{SiHa}$ and HeLa) and human embryonic kidney HeK 293 cell lines incubated with $1 \mu^{-1 m L}{ }^{-1}$ of Chitosan-NR-MSP and FAChitosan-NR-MSP. (D) Normalized mean fluorescence intensity (MFI) of Chitosan-NR-MSP and FA-Chitosan-NR$\operatorname{MSP}\left(1 \mu \mathrm{g} \mathrm{mL}^{-1}\right)$.
Further, it can be deduced that the uptake of FA-ChitosanNR-MSP in HeLa cells was 2-3 times higher than the uptake in $\mathrm{SiHa}$, and 8-9 times more efficient than in HeK 293. These observations suggest that the intracellular uptake of FAChitosan-NR-MSPs was more target-specific.

To further validate the uptake of FITC-labeled Chitosan and FA-Chitosan coated NR-MSPs in SiHa, HeLa and HeK 293 cells, confocal microscopy studies were performed. To assess the cellular state, nuclei were stained with diamidino-2phenylindole (DAPI). Confocal images of SiHa, HeLa and HeK 293 cells treated with Chitosan-NR-MSPs and FA-ChitosanNR-MSP $\left(1 \mu \mathrm{g} \mathrm{mL}^{-1}\right)$ show that the Chitosan-NR-MSPs were localized in the cytoplasm, while the FA-Chitosan-NR-MSP accumulated more closely around the nucleus (Fig. S7† and Fig. 5). The fluorescence intensity of FA-Chitosan-NR-MSP around the nucleus was increased at $24 \mathrm{~h}$ compared to $4 \mathrm{~h}$. We believe that higher FA-Chitosan-NR-MSP uptake in HeLa and $\mathrm{SiHa}$ cells would be due to FR-mediated endocytosis. These results are consistent with the cellular uptake of FA-conjugated Chitosan-NR-MSP observed in the FACS results, thus further indicating that the cellular uptake of the presented system can be enhanced by using FA as targeting ligand.
Control

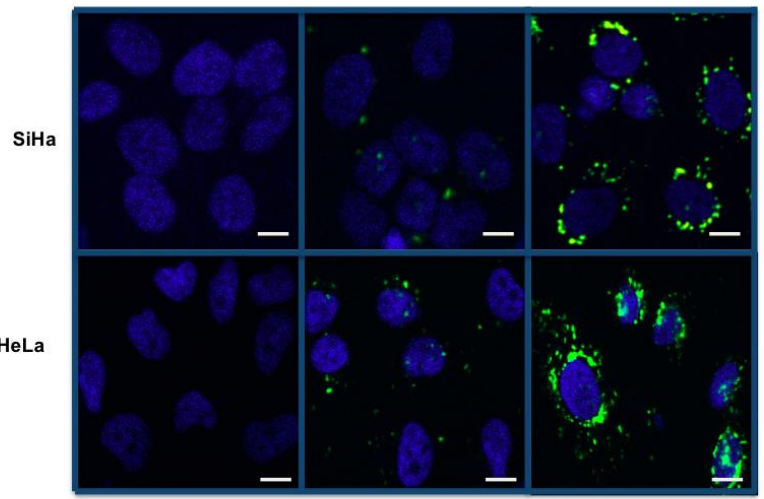

Fig. 5 Confocal microscope images showing the intracellular uptake of FITC-labelled Chitosan-NR-MSPs (green) in $\mathrm{SiHa}$ and HeLa cell lines incubated for $24 \mathrm{~h}$, shows that ChitosanNR-MSPs were localized in the cytoplasm while the FAChitosan NR-MSP accumulated more closely around the nucleus in SiHa and HeLa. Nuclei were stained with DAPI (blue). Scale bar: $10 \mu \mathrm{m}$

Assessment of cell death by caspase-3 activation assay and western blot analysis

Apoptosis is an active, genetically regulated form of programmed cell death and caspase- 3 is a cysteine protease that play a key role in the execution of the apoptotic programme. ${ }^{39}$ When we have studied the apoptotic mechanism of free AA in $\mathrm{SiHa}$ cells, we were able to demonstrate that AA primarily activates the apoptotic machinery of caspases through the intrinsic, mitochondrial apoptosis pathway. ${ }^{11}$ In order to examine the efficacy of FA- Chitosan-NR-MSP loaded with AA, we first performed caspase-3 PE staining to assess whether a lower dose of chitosan-coated AA loaded NR-MSP (Chitosan-NR-MSP/AA) would enhance apoptotic death of 
SiHa cervical cancer cells. Caspase-3 activation in SiHa cells incubated with Chitosan-NR-MSP/AA and free AA was markedly increased ( 2.5 fold) in $\mathrm{SiHa}$ cells exposed to AAloaded FA-conjugated Chitosan-NR-MSPs as compared to induction by free AA at corresponding AA concentration (Fig. $6)$.
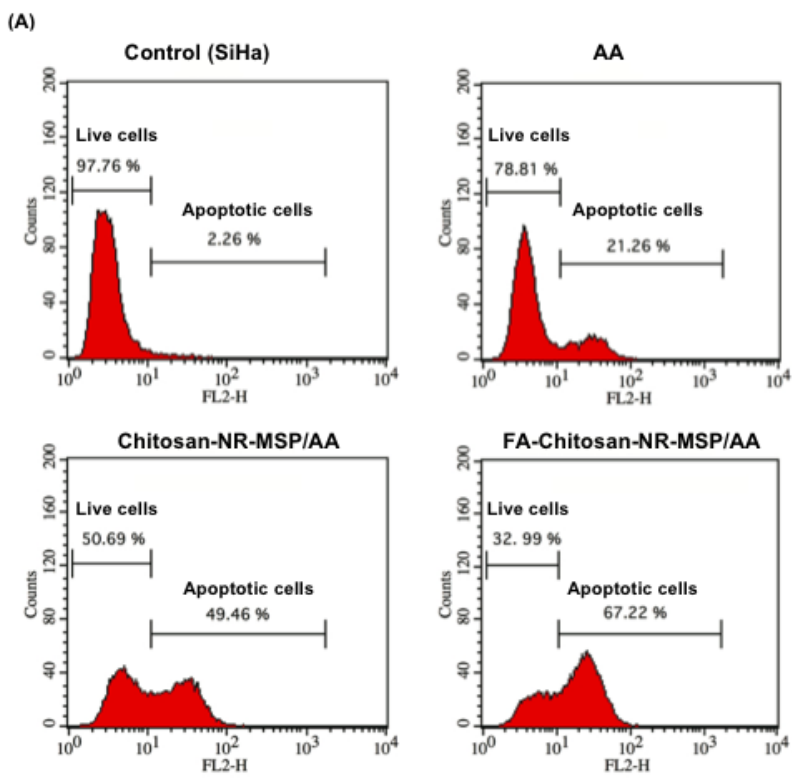

(B)

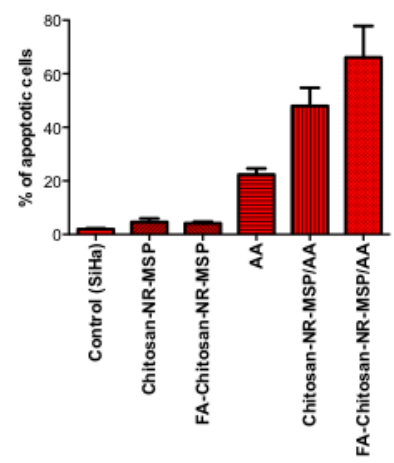

Fig. 6 (A) The cells were incubated with $20 \mu \mathrm{M}$ free AA and AA-loaded Chitosan-NR-MSP at corresponding AA concentration for $24 \mathrm{~h}$. The percentage of cells that contained activated caspase- 3 was determined as a measure of apoptosis. (B) Percentage of apoptotic cells are indicated as the proportion of cells that contained caspase- 3 activated cells.

To further confirm the effect of AA-loaded Chitosan-NRMSPs for the observed increased apoptosis on SiHa cells, we also investigated the proteolytic cleavage of procaspase- 3 and PARP by western blot analysis. PARP is a nuclear enzyme implicated in DNA repair and the cleavage of PARP has been considered to be a hallmark of apoptosis. ${ }^{40} 20 \mu \mathrm{M}$ free AA and the same amount of AA $(20 \mu \mathrm{M})$ loaded in Chitosan-NR-MSPs induced the cleavage of procaspase 3 into its active $17 \mathrm{kDa}$ fragment and the cleavage of PARP polypeptide from $116 \mathrm{kDA}$ into its characteristic $85 \mathrm{kDA}$ fragment after $24 \mathrm{~h}$ treatment (Fig. 7), clearly confirming activation of the apoptotic machinery.

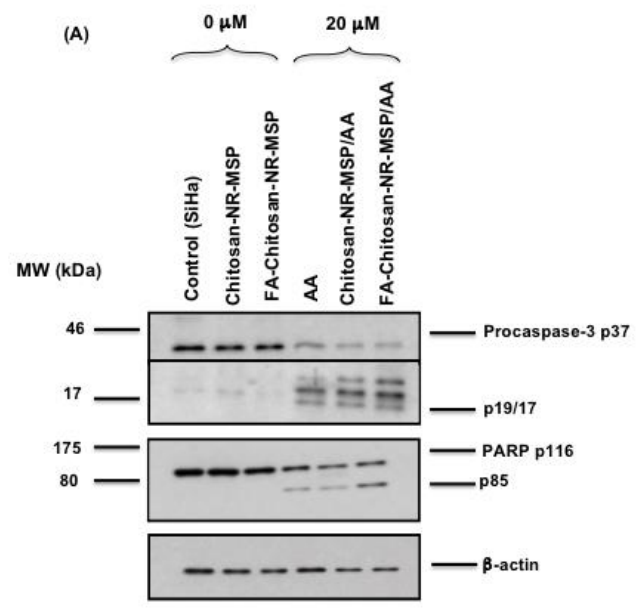

(B)

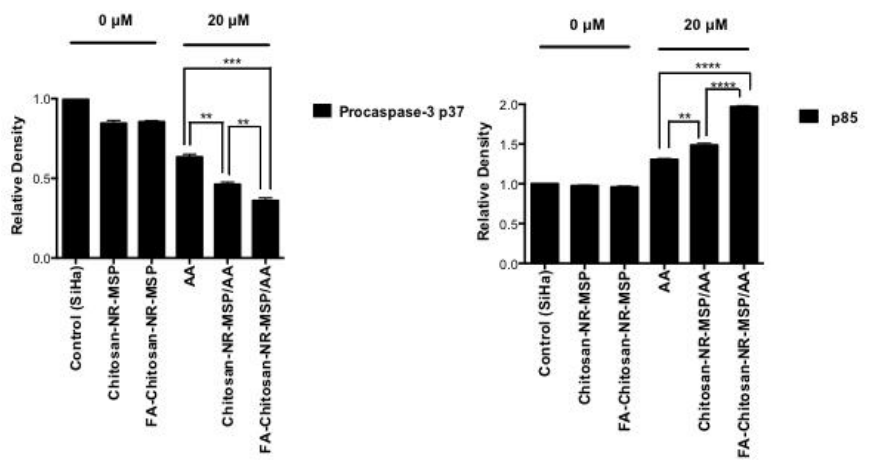

Fig. 7 (A) Western immunoblot of caspase-3 and PARP protein expression in SiHa cells. The cells treated with $20 \mu \mathrm{M}$ of AA loaded Chitosan-NR-MSPs and free AA for $24 \mathrm{~h}$. $\beta$-actin was used as a loading control. (B) The images are representative of at least three independent experiments (Mean \pm SEM; $n=3$; $* * * * \mathrm{P} \leq 0.0001, * * * \mathrm{P} \leq 0.001, * * \mathrm{P} \leq 0.01)$.

The immune blot shows that Chitosan-NR-MSPs itself did not cause cleavage of procaspase and PARP, whereas the cells treated with FA-Chitosan-NR-MSPs loaded with AA exhibited an enhanced proteolytic cleavage of intact PARP into cleaved PARP over the treatment with free AA and AA-loaded Chitosan-NR-MSP.

Previously, we have shown that only $40 \mu \mathrm{M}$ AA induces caspase 3 and PARP cleavage effectively, ${ }^{10}$ whereas in the current study, we observed that $20 \mu \mathrm{M}$ of AA loaded within FAChitosan-NR-MSPs induced effective caspase 3 and PARP cleavage. From these results, it can be concluded that the efficacy of AA could indeed be enhanced by NR-MSP formulation with retained target activity, and the efficacy could be further enhanced by FA-targeted delivery by the NR-MSPs.

\section{Conclusions}

Our previously developed mesoporous silica nanorod (NRMSP) platform was here modified with chitosan layers for the dual purpose of both pore capping as well as taking advantage of the known $\mathrm{pH}$-responsiveness of chitosan in providing enhanced drug retention under extracellular conditions. Moreover, both the rod shape of the particles as well as the 
cationic charge provided by the chitosan coating were utilized and combined in this novel nanocarrier design in order to maximize cellular uptake in the target cells. The carrier system was further tagged with FA in order to enhance the affinity towards specifically cancer cells, in the present case cervical cancer. The carrier system was further loaded with the naturally derived compound AA, a plant extract previously explored for its apoptotic activity, with the aim of increasing the target apoptosis by particle-mediated delivery. When loaded into the NR-MSPs, the apoptotic efficacy was clearly enhanced over the effect of free AA, and moreover, this effect could be further enhanced by the tagging of FA-ligands to the particles for targeted delivery. Importantly, the AA also retained its target activity upon particle loading and intracellular release. Taken together, our results show that the developed carrier could effectively be taken up by the targeted cancer cells by FAconjugation and further enhance the targeted action of AA by particle-mediated delivery utilizing NR-MSPs as carrier systems.

\section{Acknowledgements}

Sigrid Juselius Foundation (R.S.), Center of Excellence for Functional Materials (D.S.K.), Nanolith Sverige AB (E.B. \& M.O.), Academy of Finland (project decisions \#140193, \#260599 and \#278812, J.M.R.) are acknowledged for financial support. Linus Silvander is acknowledged for SEM imaging.

\section{Notes and references}

a Department of Biosciences, Cell biology, Åbo Akademi University, Tykistokatu 6B, FI-20520 Turku, Finland. Email: john.eriksson@abo.fi.

$b$ Laboratory for Physical Chemistry, Åbo Akademi University, Porthansgatan 3-5, FI-20500 Turku, Finland. Email: jerosenh@abo.fi

${ }^{c}$ Nanostructured Materials Division, Department of Physics, Chemistry and Biology, Linköping University, SE-581 83, Sweden

Electronic Supplementary Information (ESI) available: [details of any supplementary information available should be included here]. See DOI: $10.1039 / \mathrm{b} 000000 \mathrm{x} /$

1 X. Wen, D. Li, Y. Zhang, S. Liu, L. Ghali and R. Iles, Anticancer Drugs, 2012, 23, 280.

2 W.J. Qiao, H.Y. Cheng, C.Q. Li, H. Jin, S.S. Yang, X. Li, and Y.Y Zhang, Asian Pac. J. Cancer Prev., 2011, 12, 99.

3 S. Fulda, Planta Med., 2010, 76, 1075.

4 H. Itokawa, S. L. Morris-Natschke, T. Akiyama and K. H. Lee, J. Nat. Med., 2008, 63, 263.

5 K. H. Lee, J. Nat. Med., 2010, 73, 500.

6 M. S. Butler, Nat. Prod. Rep., 2005, 22, 162.

7 K. Purushothaman, R. Rao and K. Kalyani, Indian J Chem., 1975, 13, 1357.

8 P. Paul, R. Padmapriya, V.S. Periasamy, A. Riyasdeen, S. Srinag, K. Krishnamurthy, A. A. Alshatwi and M. A. Akbarsha, J. Pharmacol Pharmacother., 2012, 3, 26.

9 P. Paul, A. A. Alshatwi and M. A. Akbarsha, Sci. Pharm. Commun., 2013, 81, 559.

10 P. Paul, R. Senthilkumar, E. Peuhu, A. A. Alshatwi, M. A. Akbarsha and J. E. Eriksson, Biochem. Pharmacol., 2014, 89, 171.

11 D. Peer, J. M. Karp, S. Hong, O. C. Farokhzad, R. Margalti and R. Langer, Nat. Nanotech., 2007, 2, 751.
12 B. V. Bonifácio, P. B. Silva, M. A. S. Ramos, K. M. S. Negri, T. M. Bauab and M. Chorilli, Int. J. Nanomed., 2014, 9, 1.

13 J. M. Rosenholm, C. Sahlgren and M. Lindén, Current Drug Targets, 2011, 12, 1166.

14 J. Lu, M. Liong, Z. Li, J. I. Zink and F. Tamanoi, Small, 2012, 6, 1794.

15 M. E. Pearce, J. B. Melanko and A. K. Salem, Pharm. Res., 2007, 24, 1.

16 S. Giri, B. G. Trewyn, M. P. Stellmaker and V. S. -Y. Lin, Angew. Chem. Int. Ed., 2005, 44, 5038.

17 D. Sen Karaman, D. Desai, R. Senthilkumar, E. M. Johansson, N. Råtts, M. Odén, J. E. Eriksson, C. Sahlgren, D. M. Toivola and J. M. Rosenholm, Nanoscale Res. Lett., 2012, 7, 358.

18 V. P. Torchilin, Adv Drug Deliv. Rev., 2006, 58, 1532.

19 V. R. Sinha and A. Trehan, J. Control Release, 2003, 90, 261.

20 M. Hu, Y. Li, E. A. Decker, H. Xiao and D. J. McClements, J. Agric. Food Chem., 2010, 58, 1283.

21 A. Popat, J. Liu, G. Q. Lu and S. Z. Qiao, J. Mater. Chem., 2012, 22, 11173.

22 X. Hu, Y. Wang and B. Peng, Chem. Asian J., 2014, 9, 319.

23 J. J. Wang, Z. W. Zeng, R. Z. Xiao, T. Xie, G. L. Zhou, X. R. Zhan and S. L. Wang, Int J Nanomedicine., 2011, 6, 765.

24 V. Bansal, P. K. Sharma, N. Sharma, O. P. Pal and R. Malviya, Adv. Biol. Res., 2011, 5, 28.

25 A. Venkataraman, J. L. Hedrick, Z. Y. Ong, C. Yang, P. L. Rachel Ee, P. T. Hammond and Y. Y. Yang, Adv. Drug Delivery Rev., 2011, 63, 1228.

26 S. E. A. Gratton, P. A. Ropp, P. D. Pohlhaus, J. C. Luft, V. J. Madden, M. E. Napier and J. M. DeSimone, Proc. Natl. Acad. Sci., 2008, $\mathbf{1 0 5}, 11613$.

27 C. Guo and R. A. Gemeinhart, Eur. J. Pharm. Biopharm., 2008, 70, 597.

28 S. J. Yang, F. H. Lin, K. C. Tsai, M. F. Wei, H. M. Tsai, J. M. Wong and M. J. Shieh, Bioconjugate Chem., 2010, 21, 679.

29 E. M. Johansson, M. A. Ballem, J. M. Córdoba, M. Odén, Langmuir, 2011, 27, 4994.

30 J. P. López-Alonso, F. Diez-Garcia, J. Font, M. Ribó, M. Vilanova, J. M. Scholtz, C. González, F. Vottariello, G. Gotte, M. Libonati and D. V. Laurents, Bioconjugate Chem., 2009, 20, 1459.

31 N. Nakajima and Y. Ikada, Bioconjugate Chem., 1995, 6, 123-130.

32 U. K. Laemmli, Nature, 1970, 227, 680.

33 A. R. Dudhani and S. L. Kosaraju, Carbohydr. Polym., 2010, 81, 243. 34 N. F. Cover, S. Lai-Yuen, A. K. Parsons and A. Kumar, Int. J. Nanomedicine, 2012, 7, 2411.

35 P. J. VandeVord, H. W. T. Matthew, S. P. DeSilva, L. Mayton, B. Wu and P. H. Wooley, J. Biomed. Mater Res., 2002, 59, 585.

36 S. Naahidi, M. Jafari, F. Edalat, K. Raymond, A. Khademhosseini and P. Chen, J. Control Release, 2013, 166, 182.

37 J. Davda, and V. Labhasetwar, Int. J. Pharm., 2002, 233, 51-59.

38 J. R. Masters, Nature Reviews Cancer, 2002, 2, 315.

39 E. S. Alnemri, D. J. Livingston, D. W. Nicholson, G. Salvesen and N. A. Thornberry, Cell, 1996, 87, 171..

40 P. J. Duriez and G. M. Shah, Biochem. Cell Biol., 1997, 75, 337. 